\title{
Alchimie de la scène et précipitation des temps (à propos de Forêts de Wajdi Mouawad)
}

\section{Eric Eigenmann}

\section{(2) OpenEdition \\ 1 Journals}

\section{Édition électronique}

URL : http://journals.openedition.org/edl/1086

DOI : $10.4000 /$ edl. 1086

ISSN : 2296-5084

\section{Éditeur}

Université de Lausanne

\section{Édition imprimée}

Date de publication : 15 mars 2018

Pagination : 99-112

ISBN : 978-2-940331-67-3

ISSN : 0014-2026

\section{Référence électronique}

Eric Eigenmann, «Alchimie de la scène et précipitation des temps (à propos de Forêts de Wajdi Mouawad) », Études de lettres [En ligne], 1 | 2018, mis en ligne le 15 mars 2020, consulté le 15 décembre 2020. URL : http://journals.openedition.org/edl/1086 ; DOI : https://doi.org/10.4000/edl. 1086 


\section{ALCHIMIE DE LA SCÈNE ET PRÉCIPITATION DES TEMPS (À PROPOS DE FORETTS DE WAJDI MOUAWAD)}

Deux tableaux de Forêts, pièce de Wajdi Mouawad (2009), sont interrogés ici sous l'angle proposé par le colloque «Le penser de la scène». Après avoir montré comment, à transgresser les conventions théâtrales traditionnelles, ces tableaux déjouent les attentes en déstabilisant notamment la perception de la temporalité diégétique, cet article dégage l'exceptionnalité d'instants de théâtre qui précipitent, au sens temporel et moléculaire, des fragments de vies distinctes dans le temps et l'espace, et qui ouvrent la perspective d'une communion sensorielle paradoxalement partagée entre personnages, comédiens et spectateurs. Ces observations seront finalement liées au jeu collectif avec la contingence et la finitude de la vie humaine que constitue l'événement théâtral.

Tous les vrais alchimistes savent que le symbole alchimique est un mirage comme le théâtre est un mirage. Et cette perpétuelle allusion aux choses et au principe du théâtre que l'on trouve dans à peu près tous les livres alchimiques, doit être entendue comme le sentiment (dont les alchimistes avaient la plus extrême conscience) de l'identité qui existe entre le plan sur lequel évoluent les personnages, les objets, les images, et d'une manière générale tout ce qui constitue la réalité virtuelle du théâtre et le plan purement supposé et illusoire sur lequel évoluent les symboles de l'alchimie ${ }^{1}$.

I. A. Artaud, «Le théâtre et son double», p. 532 sq. 


\section{Troubles dans la temporalité}

Dans Forêts, troisième texte de la tétralogie publiée par Wajdi Mouawad en 2009 sous le titre Le Sang des promesses, on lit le passage suivant:

ODETTE. Edmond? Qu'est-ce que tu fais ici, tout seul, entre les pattes de la girafe?

EDMOND. La girafe et moi nous attendons la pluie. Edgar et Hélène m'ont dit que quand il pleut, la girafe est heureuse et moi, j'aime quand la girafe est heureuse.

Pluie 2006. Pluie 1943. Pluie 1873.

LOUP. Bon, ça y est...

LUDIVINE. Vous entendez?

EDMOND. Enfin!

LOUP, ODETTE et LUDIVINE. Il pleut ${ }^{2}$.

A priori énigmatique, la didascalie renvoie aux trois situations d'énonciation différentes qui déterminent les répliques, apparemment toutes en dialogue, de Loup (en 2006 à Metz), de Ludivine (dans la même ville en 1943), d'Edmond et Odette enfin (en $1873^{3}$ dans la forêt des Ardennes). Comme Edmond le fait à sa mère Odette, Loup et Ludivine avaient annoncé la pluie: «On devrait partir d'ici, le ciel se couvre, il va sûrement pleuvoir", "Regardez, il va bientôt pleuvoir " ${ }^{4}$; elles la constatent maintenant à l'unisson avec Odette dans la dernière réplique, bien que cent trente-trois ans séparent l'observation de l'aînée de celle de la cadette.

Le lecteur de la pièce a certes été initié à ce mode de dialogue lors d'un passage similaire. En l'occurrence, c'est une sonnerie de cloches qu'annonçait Edmond:

EDMOND. Alors les cloches de la cathédrale vont bientôt sonner.

2. W. Mouawad, Forêts, p. 105-109. Une première version de ce texte a été publiée en 2006.

3. Il s'agit en réalité de 1874 , si l'on en croit la précision donnée par Douglas Dupontel quelques répliques plus haut: "Albert [mari d'Odette et père d'Edmond] s'installera avec sa famille dans un domaine au milieu de la forêt des Ardennes en 1874» (Ibid., p. 108). Mais ce léger flou temporel ne porte à aucune conséquence.

4. Ibid., p. 105 sq. 
$[\ldots]$

ALBERT. [...] (Des cloches carillonnent). Ecoute. Les cloches de la cathédrale. Elles sonnent comme le signe d'une vie future qui sera belle, légère et splendide.

Un téléphone sonne à l'hospice à Matane.

Luce répond.

LOUP. Luce, c'est moi, c'est Loup! Ecoute, ce sont les cloches de la cathédrale de Metz.

Odette et Albert écoutent les cloches.

Edmond et Ludivine écoutent les cloches.

Loup et Luce écoutent les cloches 5 .

Les trois couples décrits ci-dessus sont déjà apparus tour à tour, mais isolément, chacun dans leur époque, à des stades chronologiquement un peu antérieur de leur histoire ${ }^{6}$. Si tous les personnages se trouvent cette fois au même endroit, à Metz, le téléphone de Loup à sa grand-mère Luce distend l'espace représenté dans la mesure où celle-ci participe à l'action à Matane, au Québec.

Mon interrogation découle de la réception de ces deux passages, telle que je l'ai observée lors de récentes représentations de Forêts ${ }^{7}$. Soir après soir, l'attention du public confinait en cette double occasion à une forme de sidération. Comment l'expliquer? Dans la perspective du présent colloque, j'avancerai l'hypothèse que cette réaction procède de l'expérience d'une transgression de la convention théâtrale commune sur le plan de la temporalité, soit d'une extension de potentiel surprenante par rapport $\mathrm{au}$ «penser de la scène» dont le public entretient encore majoritairement, bien que de manière impensée, la conception. En tant qu'elle "pense" son objet, l'action scénique traditionnelle réalise en effet des opérations temporelles dont il faut commencer par rappeler les termes et les enjeux.

5. Ibid., p. 97-100.

6. Soit en 1871-72, 1943 et 2006, respectivement.

7. Représentations données par l'ATDF/Atelier théâtre du Département de français de l'Université de Genève en avril-mai 2016, au Théâtre Cité bleue et au Théâtre de la Comédie à Genève lors de la soirée d'ouverture du festival Commedia (Scénographie N. Jaquerod, lumières Cl. Firmann, mise en scène E. Eigenmann assisté de S. Jehan, jeu D. Auberson-Lavoie, M. Coquillat, Th. Cornuz, L. Deconche, D. El Ariss, C. Fellrath, L. Pralong, A. Rey, L. Rodrigues, Ch. Roux, Ch. Seoane, F. Tankut, D. Vicente, G. Yurtseven, Ch. Zufferey). 


\section{La représentation démultipliée, l'accommodation déstabilisée}

Selon une tradition dont la référence originaire peut être identifiée à la mimèsis aristotélicienne, mais qu'il faut se garder de faire remonter sans réserve à la Grèce antique, ni d'ailleurs à la Renaissance et au classicisme, les jeux temporels opérés par la scène théâtrale tiennent en définitive, pour la langue française, dans la notion de représentation. Si re-présentation il y a, c'est que deux présents comme deux présences s'y articulent, propres à la réalité physique de la scène d'une part, à la fiction que celleci figure d'autre part. Le temps scénique est à la fois objectif, chronométrable, continu et subjectif, tel que le spectateur le vit "en direct»; le temps fictionnel ou dramatique reste généralement plus incertain, voire discontinu, étant établi par le spectateur à partir d'éléments référentiels. Les signes du temps fictionnel sont en outre rarement temporels au premier chef, mais le plus souvent linguistiques pour les signes directs ou "dénotatifs» ${ }^{8}$, et surtout visuels (autrement dit spatiaux, à un certain degré du moins) pour les signes indirects (l'évolution, dégradation ou non, d'un espace, d'un costume, par exemple). La première temporalité est donc perçue, la seconde conçue, c'est-à-dire reconstituée par le spectateur grâce aux informations qui lui sont fournies. Dans cette dynamique, les théoriciens identifient le "temps théâtral». Pour Anne Ubersfeld, celui-ci "peut être compris comme le rapport entre l'une et l'autre» temporalité, "celle de la représentation [...] et celle de l'action représenté» ${ }^{9}$. Que cette dualité néglige le temps du scripteur, Mouawad en l'occurrence avec sa langue et ses références socio-historiques, importe peu lorsque le temps de la représentation scénique s'en rapproche. C'est évidemment le cas du spectacle que nous observons, dont le récit met de plus au premier plan la quête que mènent une jeune fille et un paléontologue présentés comme contemporains du public ${ }^{10}$.

Or l'expérience partagée de la pluie et de la sonnerie des cloches de la cathédrale, telle que Forêts la donne en spectacle, rompt avec l'attente, fût-elle confuse aux yeux du public, de la "simple» dualité temporelle caractéristique de l'art théâtral, du fait de la pluralité des temps

8. A. Ubersfeld, L'école du spectateur, p. 242.

9. A. Ubersfeld, Lire le théâtre, p. 187.

Io. Rien ne vient marquer la modeste différence de 10 ans (de 2006 à 2016) qui, pour un lecteur ou spectateur attentif, les sépare en réalité. 
représentés simultanément. Tandis que, selon ce principe duel, la fiction suit une temporalité narrative globalement linéaire, qui n'exclut pas d'éventuels désordres chronologiques, la pièce de Mouawad réunit alors en un seul tableau des personnages qui - entre les années 1870, 1943 et 2006 - s'inscrivent dans un arbre généalogique de sept générations! Composite, temporellement monstrueux en quelque sorte, l'univers diégétique y confine au fantastique ${ }^{11}$. Voilà de quoi retenir tout particulièrement l'attention des récepteurs.

Mais la dualité constitutive du théâtre, qu'elle soit d'ailleurs spatiale ou temporelle, est-elle si ancrée dans les esprits qu’elle puisse déterminer la réception du spectacle? Il serait aisé de le soutenir en arguant que cette dualité, mutatis mutandis, constitue aussi bien tous les arts narratifs, de la littérature au cinéma en passant par la bande-dessinée. Acceptons cependant l'objection, même partiellement, et nous nous apercevrons que l'horizon d'attente alternatif ${ }^{12}$ - la confusion des deux niveaux - fait en définitive le lit d'une réception convergente. En effet, selon la lecture de Denis Guénoun, «il faut [...] admettre que la mimesis aristotélicienne est relativement indifférente à l'opposition entre la figure et son référent, voire qu'elle est construite, précisément, sur cette in-différence» ${ }^{13}$, laquelle n’est pas sans perdurer, via les objectifs esthétiques classiques, jusqu'à nos jours. Il en découle une assimilation des durées scénique et fictionnelle que la narratologie de Gérard Genette, sur le strict plan du texte, formule par une équation mathématique entre temps du récit et temps de l'histoire ${ }^{14}$. Devant la représentation théâtrale, cette assimilation relève souvent de l'expérience:

II. Rappelons la mention, au cœur de l'action, d'un personnage monstrueux à valeur allégorique: «JEANNE. [...] Un être difforme et monstrueux, né sans parole et sans conscience, vit avec [notre mère] au fond de la fosse et nous interdit de l'aider. Sa voix seule est mélodieuse. C'est lui qui chante» (Forêts, p. 47).

I2. Pour employer la terminologie introduite par Hans Robert Jauss dans le domaine de la réception en littérature.

I3. D. Guénoun, Le théâtre est-il nécessaire?, p. 21.

I4. $\mathrm{TR}=\mathrm{TH}$. Formulation par dérivation puisque, dans la définition qu'il donne de la scène au chapitre "Fréquence narrative», l'auteur du Discours du récit s'attache à définir la vitesse narratologique d'un texte écrit sous forme de dialogue, non pas de sa représentation scénique: "la scène, le plus souvent "dialoguée», [...] réalise conventionnellement l'égalité de temps entre récit et histoire [...]» (Figures III, p. 129). La précision "conventionnellement" permet à Genette de faire l'impasse non seulement sur la question du débit de parole mais sur les intervalles entre les répliques, et a fortiori sur d'éventuelles 
Pour le spectateur, il arrive vite un moment où la temporalité dramatique et la temporalité scénique (celle dans laquelle il vit lui aussi, face à la représentation théâtrale) commencent à s'interpénétrer et à renforcer mutuellement leur crédibilité ${ }^{15}$.

Ainsi judicieusement nommé par Patrice Pavis, ce «moment" a pour effet de ramener la fiction au présent. A prêter son corps et sa voix au personnage, le comédien en est évidemment le principal facteur. Dans le texte écrit, grâce à l'ellipse des verbes de parole en particulier, la voix didascalique tend de même à "présentifier" les interventions dialoguées, réduisant paradoxalement l'épaisseur du dédoublement énonciatif qu'elle indique ${ }^{16}$. Anne Ubersfeld l'a relevé: "par une sorte de loi, l'absence de référent historique passé signifie le présent» ${ }^{17}$; et Goethe en a exprimé la singularité en littérature: "le poète épique rapporte l'événement comme parfaitement passé, alors que le poète dramatique le représente comme parfaitement présent» ${ }^{18}$. Cette manière illusionniste, en définitive, d'appréhender la représentation théâtrale induit pour la fiction une chronologie linéaire, homogène à celle de l'événement scénique. Les anachronies y semblent praticables par le seul biais de la narration, le cas échéant sous la forme du théâtre dans le théâtre, soit à un autre niveau de réalité.

En revanche, relève aussi Ubersfeld:

[...] la rupture de l'unité de temps contraint le spectateur à dialectiser l'ensemble de ce qui lui est proposé, à réfléchir sur l'intervalle. Ainsi, chaque fois que la scène présente un saut temporel, s'installe dans la conscience du spectateur la nécessité d'inventer le processus qui le comblera $^{19}$.

ellipses temporelles (mentionnées p. 122 sq.) entre certaines d'entre elles, qu'elles soient tolérées ou positivement exploitées comme telles, pour ménager par exemple l'unité de temps à l'échelle de la pièce.

I5. P. Pavis, L'analyse des spectacles, p. 146.

I6. Le cas contraire est littéralement exceptionnel; dans Les Dames du jeudi de Loleh Bellon par exemple: «Le téléphone sonne. Il y a presque quarante ans» (p. 48).

17. A. Ubersfeld, Lire le théâtre, p. 202.

I8. Lettre à Schiller du 23. 12. 1797, citée par D. Chaperon: "Le travail de la narration dramatique», p. 30.

19. A. Ubersfeld, Lire le théâtre, p. 193. 
C'est à fortiori l'effet que produisent les télescopages temporels de Forêts. Ils contrarient cette homogénéité entre temps de la scène et temps de la fiction, tout autant que la dualité temporelle du théâtre, invitant à reconsidérer radicalement l'intervalle - en termes d'optique: à en accommoder constamment la perception, sans parvenir en l'occurrence à la fixer.

Forêts, ou la théâtralité d'une furtive interpénétration des âges

Les précédents ne manquent pas, objectera-t-on encore. Aussi anciens que le théâtre lui-même, qu'ils soient inscrits dans le texte dramatique ou introduits à la représentation, les écarts par rapport à cette idée doublement mimétique ont connu, dès les années 1930, un développement croissant lié à de nouveaux partis pris esthétiques, notamment épiques ou performantiels. Par rapport à la temporalité du texte écrit et de la scène, qui a peu évolué au long de l'Histoire, celle de la fiction s'est autonomisée et démultipliée, mais aussi diluée au profit de l'événement vécu sur le plateau. On le sait bien, nombre de pièces sont fondées sur une analepse, telles L'Inconnue d'Arras d'Armand Salacrou (1930), Death of a Salesman d'Arthur Miller et Der Kaukasische Kreidekreis de Bertolt Brecht (1949). D'autres le sont sur une durée fictionnelle qui excède toute tolérance dans l'application de la règle classique d'unité de temps, telles A long Christmas dinner de Thornton Wilder (1931) et Le Printemps de Denis Guénoun (1985), dont les actions s'étendent respectivement sur 90 et 50 ans environ. Certaines dilatent au contraire la durée d'une action plus courte que sa représentation textuelle ou scénique, telle Intérieur de Maurice Maeterlinck (1895). Exacerbant la discontinuité spatio-temporelle, d'autres pièces encore procèdent à des collages ou à des montages de bribes de dialogues (ou monologues) appartenant à des situations différentes, telles Bonjour, là, bonjour de Michel Tremblay (1974), Portrait d'une femme de Michel Vinaver (1984) et De la Révolution de Joseph Danan (2006), texte dont la typographie en deux colonnes, parfois plus, exprime la simultanéité scénique de répliques éloignées dans le temps, celles par exemple de "CÉLIA ANNÉES 70 » et de "CATherine années 2000 ». Ailleurs, c'est le même personnage qui intervient à plusieurs étapes de sa vie - Albertine en cinq temps de Michel 
Tremblay (1984) - et dialogue parfois avec lui-même - King de Michel Vinaver (1998).

Lorsque enfin l'écriture respecte peu ou prou les unités classiques, on l'a dit, son passage à la scène ne manque pas de multiplier, sans doute de plus en plus, les références temporelles hétérogènes, sinon disparates. La mise en scène par Nicolas Stemann de Nathan le sage de Lessing, combiné à Crassier d'Elfriede Jelinek sous le titre Nathan?, en offre un exemple récent ${ }^{20}$ : une bonne part du dialogue était lue sur des lutrins par des comédiens en tenues d'aujourd'hui, ce qui n'empêchait pas l'apparition de personnages en costumes du XVIII siècle. S'y côtoyaient robes de croisé et perches à selfie. L'écart temporel était ainsi constamment maintenu concret, visible, patent entre le passé de la fiction et le présent de la représentation, soit la performance scénique proprement dite en interaction avec le public, qui s'en trouvait privilégiée. C'est là depuis une cinquantaine d'années dans l'histoire du théâtre une tendance profonde, qui rejoint en partie les aspirations d'Antonin Artaud pour une action scénique libérée de ses fonctions narratives, voire plus largement référentielles:

Ce déplacement de la normativité scénique vers la présentation sèche, vers la justesse d'un existé renvoyé à lui-même et cependant offert au regard (et à l'écoute), affecte toutes les exigences de la scène ${ }^{21}$.

Nos deux tableaux de Forêts, bien qu'ils s'inscrivent dans cette évolution formelle, sont toujours ressentis par le public comme une transgression dans son attente ordinaire d'une temporalité théâtrale linéaire. C'est qu'ils opèrent aussi à un autre niveau. Comme tous les exemples précédents, ils déjouent l'homogénéité temporelle de la scène et de la fiction et difractent dans le temps les éléments fictionnels comme autant de segments plus ou moins décousus. Mais, dès lors que tous les protagonistes, "datés" comme ils le sont chacun, accomplissent ensemble la même action - écoutent les mêmes cloches, contemplent les mêmes gouttes de pluie, également datées -, ces événements maintiennent la tension entre les différentes situations et, simultanément, abolissent à leur manière le temps comme s'ils trouaient l'espace spatio-temporel pour se faire entendre aux trois générations concernées. Il ne s'agit pas de dessiner

20. Programmé au Théâtre Vidy-Lausanne en septembre 2016.

2I. D. Guénoun, Le théâtre est-il nécessaire?, p. 148. 
une évolution, avec ses constantes et ses modifications, ni de dégager une manière d'intemporalité en résorbant les différences d'époque dans une énonciation ou une interlocution commune, mais bien de saisir un instant exceptionnel de communion paradoxale à travers les âges, entre des membres d'une famille dont toute la pièce dépeint au contraire, sur un laps de sept générations, l'impossible réunion. La nature sonore de l'événement et sa simplicité contribuent de surcroît à la participation du public, en vertu du sens de l'ouïe sollicité: également diffusé dans la salle par la bande-son, le bruit de la pluie et des cloches nous pénètre à notre tour immédiatement, sans décodage linguistique préalable.

C'est donc ce que Forêts effectue sans cesse, en reculant et en avançant par sauts dans le temps, que cet instant-là cristallise. Mieux, il précipite, au sens à la fois temporel et moléculaire, des fragments de vies distinctes dans le temps et l'espace des trois récits théâtraux entrecroisés. Evoqué par Lacan dans une autre perspective ${ }^{22}$, le point de capiton - cette suture qui, dans l'art de la matelassure, comprime en un point différentes couches de tissus - pourrait aussi servir de métaphore, imparfaite à son tour, pour illustrer cet instant. Une troisième figure s'avère cependant plus adéquate dans le contexte mémoriel de l'action imaginée par Mouawad, celle de la "madeleine de Proust», dans Du côté de chez Swann; la fameuse expérience de Marcel y est d'ailleurs comparée à une vision de théâtre ${ }^{23}$. Tout se passe en effet comme si, chez Loup, la perception des cloches de la cathédrale et de la pluie ${ }^{24}$, à Metz, réveillait une mémoire ancestrale lui permettant de remonter dans le temps pour goûter la même sensation en compagnie de ses aïeux, à l'endroit précis où ils l'ont déjà vécue - soit de retrouver, objet de sa quête, le temps perdu de ses origines.

22. J. Lacan, Le séminaire, livre III. Les psychoses, p. 303.

23. «Et dès que j'eus reconnu le goût du morceau de madeleine trempé dans le tilleul que me donnait ma tante [...], aussitôt la vieille maison grise sur la rue, où était sa chambre, vint comme un décor de théâtre s'appliquer au petit pavillon donnant sur le jardin, qu'on avait construit pour mes parents [...]; et avec la maison, la ville [...]. [T] out cela qui prend forme et solidité, est sorti, ville et jardins, de ma tasse de thé » (M. Proust, Du côté de chez Swann, p. 47 sq.). Je souligne.

24. Je n'ose parler de précipitations (météorologiques)! D’autant moins que P. Pavis a déjà exploité le jeu de mot avec bonheur, sur le même sujet théorique de surcroît. Dans son Dictionnaire du Théatre, p. 349-352, il conclut en effet par ces mots l'article qu'il consacre au "Temps»: "Du temps théâtral, on dira donc qu'il est variable, avec des risques de précipitations »... 
L'efficacité d'un tel dispositif auprès des spectateurs recèle une potentielle leçon de théâtralité. Tandis que Patrice Pavis souligne la fécondité en la matière des rapports variables qu'entretiennent le temps de la scène et le temps de la fiction ${ }^{25}$, Antoine Vitez va jusqu'à revendiquer la distance temporelle comme un principe esthétique:

Le théâtre, comme forme, est lié, je crois, plus ou moins consciemment, au Passé. On raconte l'histoire du Passé, on est habillé comme au Passé, on a un mode d'expression qui est un mode d'expression passé ${ }^{26}$.

On se rappelle par ailleurs la définition de Roland Barthes, qui conclut aux vertus, sur la scène, de la "polyphonie informationnelle», pour résumer la théâtralité en une "épaisseur de signes" ${ }^{27}$. A celle-ci contribue indéniablement la polyphonie temporelle de Forêts et de ses tableaux exemplaires en particulier. Pour paraphraser Barthes, c'est donc cela aussi, la théâtralité: une épaisseur de temps. Anne Ubersfeld parle précisément d' "épaisseur temporelle ${ }^{28}$ ", mais aussi de "profondeur":

[...] chaque fois qu'il [le metteur en scène] insiste sur la théâtralité, qu'il fait prendre conscience au spectateur de sa présence concrète au théâtre, c'est-à-dire de son temps de spectateur, la profondeur du temps fictionnel apparait ${ }^{29}$.

\section{L'expérience partagée d'une résurrection laïque}

D'où vient-il que cette profondeur temporelle là, plongeant simultanément ou presque de l'espace scénique vers des espaces diégétiques pluriels et lointains pour la plupart, trouble davantage, dans Forêts même, que son équivalent spatial? Sans doute de ce qu'elle touche à l'inexorabilité du temps humain et à sa finitude, qui en apporte l'ultime confirmation, dimension tragique qui marque la temporalité et non pas la spatialité. La représentation théâtrale détient ce privilège de donner

25. P. Pavis, «Temps», p. 349-352.

26. A. Vitez, Le théâtre des idées, p. 129.

27. R. Barthes, «Littérature et signification", p. 258.

28. A. Ubersfeld, Lire le théâtre, p. 190.

29. A. Ubersfeld, L'école du spectateur, p. 247. Je souligne. 
corps et souffle à un passé recelant une part, même minime, des origines - l'invisible par excellence - qui échappent aux spectateurs comme aux protagonistes de Forêts, et de le faire en assemblée publique, en présence de tous les participants du processus:

Le jeu du comédien est une énonciation au présent qui oblige donc le spectateur à éprouver physiquement, comme au présent, le passé fictionnel. Ce paradoxe, fondateur de la théâtralité vécue comme événement, a des conséquences tout à fait importantes: elle oblige le spectateur à éprouver comme vivant et réel un passé disparu; elle instaure donc la dialectique vécue passé-présent ${ }^{30}$.

Le théâtre se profile en ce sens comme la possible mise en spectacle d'une résurrection laïque qui, loin de l'occulter, joue de sa fictionnalité... Un tel miracle - ou mirage, dirait Artaud $^{31}$ - ne se produirait-il que l'espace d'un instant, il participe en outre d'une activité exceptionnellement cyclique: le théâtre, note Lucile Garbagnati, «c'est le paradoxe de la représentation à la fois irréversible dans son déroulement, réversible de par sa répétition ${ }^{32}$.

L'événement trouve une forme d'accomplissement dans la représentation, d'une grande fréquence dans le répertoire dramatique ${ }^{33}$, de personnages frappés par la mort, non seulement dans le cours de l'action, au dénouement des tragédies par exemple, mais aussi dès leur entrée en scène lorsque ce statut contribue à leur identité; ou d'une autre manière lorsqu'ils appartiennent à l'Histoire ou à la mythologie. Dès que l'exigence de vraisemblance s'allège, le thème de la visite aux Enfers, significativement, connaît de multiples variations, de Voyage chez les morts d'Eugène Ionesco (1980) aux Enfers ventriloques de Sylviane Dupuis (2004) ${ }^{34}$, en passant par la visite inverse, d'un «revenant» nar-

30. A. Ubersfeld, L'école du spectateur, p. 243. On ne discutera pas ici les limites de "l'obligation» décrétée par Ubersfeld.

3I. Voir la citation en exergue du présent article.

32. L. Garbagnati, "Quelques mots d'histoire», p. 12 sq.

33. Fréquence probablement comparable dans le répertoire cinématographique, mais pas dans les autres arts.

34. S. Dupuis, Les Enfers ventriloques, Comp'Act, 2004. Guidée par Shakespeare dans des enfers dantesques, une dramaturge rencontre les plus illustres auteurs, d'Eschyle à Sarah Kane, et quelques grands personnages de l'histoire du théâtre, sans oublier «l'Ombre maternelle» récemment disparue. 
rateur, dans Juste la fin du monde de Jean-Luc Lagarce (1990). Si les "disparus» occupent tant la scène, n'est-ce pas parce qu'elle exerce son pouvoir de figurer par des vivants des personnages qui ne le sont plus ou pas encore, ou qui ne le sont pas sous le même ciel? Le comédien en porte toutefois le reflet, qui le distingue du commun des mortels, comme Anne Ubersfeld l'a encore finement observé:

Et notre regard ne voit pas, ni n'entend le comédien comme un autre corps, une autre voix: ils sont vus aussi de profil comme on ne voit pas ceux qui s'adressent à nous, mais comme apparaissent ceux dont l'existence lointaine voyage inexplicablement très loin de nous - les morts par exemple ${ }^{35}$.

Les saluts au public qui, à de très rares exceptions près, marquent la fin de la représentation théâtrale n'en sont que plus remarquables par l'énergie qui s'en dégage, la troupe réunie redoublant en général de vitalité. Est éloquente à cet égard la comparaison entre cet ultime moment commun - pour lequel les morts se relèvent! - et le générique de fin au cinéma, qui affiche le plus souvent en un déroulement mécanique une simple liste d'acteurs, éventuellement décédés. Mais que saluent vraiment ces saluts aujourd'hui, où la réciprocité des applaudissements devient courante? Les observations formulées ici, à propos de l'emblématique quête de Loup sous la plume de Wajdi Mouawad comme de toute exploration théâtrale d'une profondeur temporelle, invitent à conclure qu'ils saluent également la maîtrise, partagée entre scène et salle, de la contingence lors du spectacle écoulé, voire le salut gagné de part et d'autre de la rampe, si provisoire qu'il soit, par et dans le jeu de la représentation. Car, sur le mode du jeu, dont on sait combien il contribue à construire l'être humain, l'expérience interactive de la scène, résonances proustiennes comprises, œuvre à la recherche du temps perdu - de tous les temps perdus, passés, futurs et sans doute présents. Son pouvoir de sidération s'avère d'autant plus fort qu'on y joue à prendre l'ombre pour la proie.

Éric EIgenmann

Université de Genève

35. A. Ubersfeld, L'école du spectateur, p. 343. Je souligne. 


\section{BIBLIOGRAPHIE}

Artaud, Antonin, "Le théâtre et son double», Euvres, Paris, Quarto Gallimard, 2004.

BArthes, Roland, "Littérature et signification ", in Essais critiques, Paris, Seuil, 1981 [1963], p. 258-276.

Bellon, Loleh, Les Dames du jeudi, L'Avant-Scène Théâtre, 1288 (septembre 2010).

Chaperon, Danielle, "Le travail de la narration dramatique», in Arielle Meyer MacLeod, Michèle Pralong (éds), Raconter des histoires. Quelle narration au théâtre aujourd'hui?, Genève, MetisPresses, 2012, p. 27-41.

Garbagnati, Lucile, "Quelques mots d'histoire», in Temps scientifique, temps théâtral, Besançon, Centre Régional de Documentation Pédagogique de Franche-Comté, 2001, p 9-17.

Genette, Gérard, Figures III, Paris, Seuil, 1972.

Guénoun, Denis, Le théâtre est-il nécessaire?, Paris, Circé, 1997.

LaCAN, Jacques, Le séminaire, livre III. Les psychoses, Paris, Transcriptions, $1955-56$.

Mounwad, Wajdi, Forêts. Le sang des promesses 13, Nouvelle édition, Montréal, Leméac/Babel, 2009.

Pavis, Patrice, L'analyse des spectacles, Paris, Nathan, 2003 [1996].

_, "Temps", Dictionnaire du Théâtre, Paris, Dunod, 1996, p. 349-352.

Proust, Marcel, À la recherche du temps perdu. Tome 1. Du côté de chez Swann, Paris, Gallimard, 1954.

Ubersfeld, Anne, Lire le théâtre, Paris, Messidor/Éditions sociales, 1982 [1977].

—, Lire le théâtre. Tome 2. L'école du spectateur, Paris, Messidor/Éditions sociales, 1981.

Vitez, Antoine, Le théâtre des idées, Paris, Gallimard, 1991. 
